\title{
Label free non-invasive imaging of topically applied actives in reconstructed human epidermis by confocal Raman spectroscopy
}

\author{
Franziska D. Fleischli, Stephanie Mathes, Christian Adlhart \\ Institute of Chemistry and Biological Chemistry, Zurich University of Applied Sciences, ZHAW, \\ Einsiedlerstrasse 31, 8820 Wädenswil, Switzerland \\ Christian Adlhart, E-mail: christian.adlhart@zhaw.ch
}

\begin{abstract}
Raman spectroscopy has become a versatile tool for the in vivo charaterisation of skin. Here we describe use of Raman spectroscopy for high resolution optical cross sectioning to resolve skin constituents and administered drugs at the cellular level. Percutaneous penetration is typically studied using permeation cells with biopsies of animals or human skin. Although this technique provides valuable clinical data, little insight is gained in the microstructure of drug penetration (intercellular or transcellular) or in the mode of action of applied vehicles or penetration enhancers. Therefore, a Raman microspectroscopic method was combined with a confocal scanning setup to image the microstructure of commercially available skin models (SkinEthic $®$ ) and the spatial distribution of penetrated actives. The models' microstructure was scanned without any special treatment or environment such as cutting, staining, freezing, or application of vacuum. The non-invasive Raman images reveal the layered structure of stratum corneum. This in particular for lipids while water tends to be more evenly distributed. When penetration of the hydrophilic active glycerol and the lipophilic octyl methoxycinnamate, OMC, was studied, a strong correlation between the local distribution of skin constituents and the hydrophilic / lipophilic character of the active was observed.
\end{abstract}

\section{Introduction}

Skin effectively inhibits penetration of chemicals as well as microbial invasion. In particular the outer cornified layer of the epidermis, the Stratum corneum (SC), is crucial for the barrier function of the skin. A highly ordered structure of hydrated keratin and lipid bilayers with specific variations in composition determines the barrier properties, e. g. natural moisturising factors (NMF) hold water hygroscopically in skin and an acidic $\mathrm{pH}$ on the skin surface protects against microbials ${ }^{1-4}$.

Thus topically applied drugs have to overcome the SC to penetrate into skin. Their penetration properties depend amongst others on their molecular weight and their hydrophilic/lipophilic character ${ }^{5}$. In addition, the penetration can be enhanced by vehicles added to the drug's formulation $^{6-8}$. But, accelerated penetration is not in every case desirable. While inflammation inhibitors should permeate SC as fast as possible to reach the inflamed area, UV absorbers in sun screens should remain at the surface to be effective. Therefore detailed understanding of penetration microroute and kinetics is fundamental for developing effective actives and formulations.

In many cases, a direct testing on human skin in vivo is not possible, e.g. because the used substances are critical, not yet tested or the methodology is not suitable for in vivo investigations. For in vitro studies, biopsies of human or animal skin are used as a substitute with the disadvantage that the condition of the ex vivo skin is different to the in vivo situation. In addition, access to skin biopsies is limited and the investigators are faced with interbatch variability. Therefore reconstructed skin models of human epidermis have been developed in the past decades. Skin models commercially available and are widely used for risk assessment and to 
investigate physiological processes after topical application of an active $e^{9,10}$. They are well characterized with regards to morphological appearance and lipid composition ${ }^{11}$. These skin models are an elegant tool for studying penetration and permeation of topically applied actives although the permeability is higher compared to human skin in vitro. But the ranking of compounds is similar and the data are highly reproducible ${ }^{12-14}$. The reduced barrier of skin models is mainly attributed to differences in lipid composition and lipid lamellar organization as well as the lack of desquamation ${ }^{12,15}$. Nevertheless because skin models are readily available and because inter-batch variation in composition and structure are controlled, they are a suitable alternative for penetration and permeation studies ${ }^{12,16-18}$.

Many methodologies were applied to study skin penetration and permeation. In vivo methods are in general limited by measuring physical properties of the SC barrier such as impedance, water content or water loss of the skin rather than directly tracking the active compound ${ }^{19}$. By tape stripping, penetration profiles of topically applied actives could be determined, but the active concentration is usually averaged per layer and depth ${ }^{20,21}$. The permeation coefficients of topically applied actives are mostly determined in vitro using diffusion cells. Both biopsies (human and animal skin) and reconstructed skin equivalents were characterized ${ }^{14,22,23}$. These experiments provide information on permeation kinetics and quantity as well as on the effect of the formulation. In addition, the amount of penetrated actives within the skin layers is quantifiable by separating SC, epidermis and dermis.

We were interested in the skin's reaction at the early stages of penetration. In addition, the skin sample should be in a state as natural as possible. Therefore we applied confocal Raman microspectroscopy, a non-invasive and label free method that provides spatially resolved information about the chemical composition. Raman spectroscopy is a strong technique for analysing biological samples in general ${ }^{24}$ and the penetration, also time and spatially resolved, in particular $^{25-29}$. By focussing the excitation laser into the skin sample, penetration of actives is tracked in microscale. Furthermore, confocal Raman microspectroscopy allows following changes in the molecular composition of the skin during the penetration. This method is even applicable for human skin in vivo, e.g. for measuring the water content, depth profiles of skin components or topically applied compounds ${ }^{2,28,30}$. Raman spectroscopy was also applied to skin models, but without resolving their heterogeneous cellular microstructure ${ }^{31}$.

Confocal Raman microspectroscopy has the potential in providing information on the microstructure of organotypic skin models upon penetration of topically applied actives to deepen our understanding in microroute of drug penetration. Because the entire skin sample is characterised, the water content can be determined. This is not possible for other imaging methods which require special sample treatment or environment such as cutting, staining or labelling and freezing or application of vacuum (e.g. immune histology, fluorescence microscopy and two-photon imaging) or only sections can be analysed (e.g. energy dispersive X-ray and IR spectroscopy) ${ }^{20,32-37}$.

In this study, we analysed the penetration of topically applied actives into reconstructed human epidermis by confocal Raman microspectroscopy. We were interested in the penetration microroute at the cellular level together with the chemical composition and structure of the epidermal model. Raman based imaging techniques were applied to the skin model and distribution maps of skin components and the applied actives were extracted. First, the composition and microstructure of untreated skin models was analysed with special focus on water and lipid concentration. Then, the penetration of a hydrophilic and a lipophilic active (glycerol and octyl methoxy cinnamate, OMC, respectively) was monitored. 


\section{Materials and Methods}

\subsection{Skin models}

Reconstructed human epidermal (RHE) skin substitutes were purchased at SkinEthic $\circledast$ Laboratories, Lyon, France. There, keratinocytes are cultured on a polycarbonate filter. The epidermis is established by the differentiation of keratinocytes at the air-liquid phase. After 11 days of growth a functional barrier namely the Stratum corneum is formed. We received the skin models at day 18. Three batches of RHE containing 12 RHE models each were investigated and various formulations were applied. The RHE models were kept in an incubator for $18 \mathrm{~h}\left(37^{\circ} \mathrm{C}, 5\right.$ $\% \mathrm{CO}_{2}$ ) according to the recommendation of SkinEthic $\AA^{10,38}$. Afterwards, the RHE models were used for the Raman experiments within three days. The typical thickness of the SC at the days of experiment was 50 to $60 \mu \mathrm{m}$.

Cryo sections were made to get an overview of the skin structure. They were stained by nile- red (lipid staining). As skin model Episkin from SkinEthic $®$ was used. It is constructed on a collagen gel and shows a similar SC-epidermis structure to RHE. A stock solution was prepared, which contained $0.05 \%(\mathrm{wt} / \mathrm{vol})$ Nile red (Sigma) in acetone and was stored at $4{ }^{\circ} \mathrm{C}$. For staining the stock solution was diluted with the same volume of a mixture of glycerol / water in a ratio of 75:25 ( $\mathrm{vol} / \mathrm{vol})$ to a final concentration of $2.5 \mathrm{mg} / \mathrm{ml}$. A drop of the dye solution was applied to each section and immediately covered with a coverslip. Nile red fluorescence was captured with a Zeiss microscope equipped with a fluorescein $(450-490 \mathrm{~nm})$ excitation filter.

\subsection{Formulations}

The actives were applied as solutions. Glycerol, octyl methoxycinnamate (OMC) and ethanol were purchased at Fluka/Sigma Aldrich. Glycerol and OMC were dissolved in ethanol at room temperature; the concentration was $50 \mathrm{wt} \%$ and $1 \mathrm{wt} \%$, respectively. This difference in concentration is on one hand related to the low solubility of OMC in ethanol and on the other hand on the low intensity of the Raman signal of glycerol. But it was expected from literature that OMC penetrates slower than glycerol and therefore the chosen concentrations are high enough for maintaining reservoir conditions at the skin surface during application ${ }^{5,39}$.

\subsection{Topical application}

The skin samples were removed from the incubator $1 \mathrm{~h}$ before application. To apply the solutions, the skin models were removed from the medium, washed with distilled water and carefully

dabbed. The solution was applied directly on the surface $\left(20 \mu \mathrm{cm}^{-2}\right)$. After $15 \mathrm{~min}$, the supernatant solution was removed. The skin models were cut out of the inset and immediately placed into a sealed sample holder to avoid drying-out. Raman spectra were acquired right thereafter.

\subsection{Confocal Raman spectroscopy and spectral fitting}

The Raman spectra were acquired with an alpha 300R Raman microscope (WITec, Ulm, Germany) equipped with a $532 \mathrm{~nm}$-excitation laserand a piezo scanning table for threedimensional scanning. The sample holder was sealed with a microscope cover glass (Menzel, 0.13-0.16 $\mathrm{mm}$ ) and the skin's surface was in direct contact with the cover glass. Spectra were acquired through the cover glass using a 100x/1.25 oil-immersion objective and immersion oil $\left(n_{\mathrm{D}}\right.$ 
$=1.515)$. Virtual cross sections were obtained by mapping an area of $50 \mu \mathrm{m}$ in width and up to 50 $\mu \mathrm{m}$ in depth $\left(0.5 \mu \mathrm{m}\right.$ step size, $0.2 \mathrm{~s}$ integration time per spectrum, spectral range $0-4000 \mathrm{~cm}^{-1}$, spectral resolution $\left.4 \mathrm{~cm}^{-1}\right)$. The laser power $(<30 \mathrm{~mW})$ was adjusted for each measurement. Since the skin samples were humid, there were particular difficulties in preventing them from thermal degradation. However, thermal degradation happened in some experiments, which were then discarded and repeated. We used a fitting procedure based on reference spectra similar to Caspers et al. ${ }^{26}$, but we increased the fitting range to 400 to $3800 \mathrm{~cm}^{-1}$, which allows the simultaneous determination of water $\mathrm{OH}$-stretching vibrations $\left(3300 \mathrm{~cm}^{-1}\right.$ to $\left.3700 \mathrm{~cm}^{-1}\right)$ and fingerprint and functional group peaks from the actives and the skin's matrix $\left(400 \mathrm{~cm}^{-1}\right.$ to 1800 $\left.\mathrm{cm}^{-1}\right)$.

For each component, a local concentration coefficient $c_{i}$ was obtained by a fitting process. The Raman spectrum of a mixture $R$ can be expressed as the sum of component Raman spectra $R_{i}$ multiplied with their concentration coefficients $c_{i}$ :

If the Raman spectra are represented as vectors, the fitting coefficients can be calculated by a matrix division according to:

where $\vec{c}$ is a vector containing all coefficients and $A$ the matrix containing all reference spectra. The coefficients $c_{i}$ of an entire optical cross sectioning are then displayed in a distribution map. We used the following spectra as references: keratin (as representative of all proteins), lipids (average spectrum extracted from RHE model measurements), water, glycerol, OMC and ethanol as well as a spectrum from the cover glass. The fitting program was created with the built-in software of the Raman instrument. Because the Raman signal in general decreases by focussing in a semi-transparent sample, all coefficients are normalized with the Keratin coefficient at the same position. It is worth to note that the Keratin content is not homogeneous within epidermis but it is the component in the skin which is ubiquitous in considerable concentration ${ }^{24}$. Distribution maps were similar between different RHE models, therefore only one example is shown for each applied active.

\section{Results and Discussion}

Confocal Raman microspectroscopy was used for high resolution optical cross sectioning of skin to resolve skin constituents and topically administered drugs simultaneously and at the cellular level.. This means that changes in the skin structure and/or composition can be directly related with the penetration behaviour of the penetrating compound.

\subsection{Untreated samples}

Before the penetration of any active was analysed, untreated RHE samples were studied. A Raman spectrum close to the surface is given in Fig. 1. Virtual cross sections were made of the $\mathrm{SC}$ of skin models by imaging with confocal Raman microspectroscopy. The distribution map of a particular component is constructed by displaying the fitting coefficients of the corresponding reference relative to the position of moving microscope stage. In this way, a distribution map for each reference was extracted, that corresponds to the relative concentration of the compound of interest. Water and lipid distribution of the untreated skin model are shown in Fig. 2. The lipid distribution is resolved at the cellular level and a fine structure corresponding to the layered structure of the skin model is observed: we find thin lipid layers and micron-sized lipid droplets 
(see Fig. 2 middle). The layers are mainly parallel to the skin surface and occasionally, they show kinks. The lipid stained biopsy shows the same structural features (see Fig. 2 right). Water has a complimentary distribution to lipids, i.e. a low local water concentration is observed at spots with high lipid concentration and vice versa.

There are important differences between the here investigated RHE models and the human in vivo situation. First, the thickness of the $\mathrm{SC}$ is different and with 50 to $60 \mu \mathrm{m}$ thickness in RHE significantly larger than typical values for the human volar forearm with 10 to $20 \mu \mathrm{m}$. This, because the SC is growing continuously in RHE models due to the lack of desquamation. Second, the water content in RHE models is higher than in the human in vivo situation (approximately $70 \mathrm{wt} \%$ compared to typically $25-35 \mathrm{wt} \%$ ). The higher water content is possibly a result of the high humidity conditions given during the incubator cultivation. A water gradient similar to the human skin in vivo is not observed for skin models ${ }^{27}$. Third, the ceramide content is higher in the SC of human skin compared to the epidermal layers, while for RHE, the ceramide content fluctuates around a high value. Forth, we did not observe any natural moisturizing factors (NMF) within the RHE models by Raman spectroscopy, while NMF molecules are detectable by Raman spectroscopy for human skin in vivo ${ }^{2}$. These differences may explain the different barrier properties of skin models and human skin, which have already been outlined in several investigations $^{14,40}$.

\subsection{Glycerol, a hydrophilic active}

As hydrophilic active, glycerol, a skin moisturizer, was analysed (water-octanol partition coefficient log $\left.K_{\mathrm{ow}}=-1.76\right)^{11}$. The peaks of glycerol appear in the Raman spectrum of treated RHE models (see Fig. 1). The distribution maps of glycerol, lipids and water show that glycerol concentration is high in the water-rich areas and low in the lipid-rich areas (see Fig. 3). This is expected for a hydrophilic species. Glycerol led to a swelling of the water-rich domains. Thus the lipid layers are more separated than in the untreated skin model.

\subsection{Octyl methoxycinnamate (OMC), a lipophilic active}

As hydrophobic active, we chose octyl methoxycinnamate (OMC) which is a UV absorber that is found in many sun screen formulations (water-octanol partition coefficient $\log K_{\mathrm{ow}}=+6.1$ ). The peaks of OMC appear in the Raman spectrum of treated skin (see Fig. 1). Even at low concentration, the Raman spectrum is dominated by the OMC due to its high Raman activity. The distribution maps show that OMC is accumulated at the skin's surface and has only penetrated a short distance into the SC within the 15 min of application (see Fig. 4). The local concentration of $\mathrm{OMC}$ varies within equal depth. The areas of highest concentration correspond to lipid droplets in the lipid distribution map (see marks in Fig. 4), i.e. OMC is concentrated in the lipid-rich area.

The coefficients of the actives correlate with the water coefficients (see Fig. 5). For OMC treated RHE models, the OMC coefficients show a negative correlation with the water coefficients. That affirms the conclusions drawn from the distribution maps, mainly preference of OMC for the lipid phase rather than the aqueous phase. In contrast, the glycerol coefficients correlate positively with the water coefficients for glycerol treated RHE models, as expected from the hydrophilic character of glycerol.

\section{Conclusion}

We developed a Raman based method to visualize the distribution of lipids, water, and topically applied actives in reconstructed human epidermal models. By applying this confocal Raman 
microspectroscopic approach, we analysed the penetration of a hydrophilic and a lipophilic active into the RHE models. In untreated RHE models the water content is high throughout the entire SC. Typical water gradients as for human skin in vivo, starting with a low water content at the SCs surface that increases down to the epidermis, are not observed for the RHE models. In addition to the lipid layers that are also observed in SC, lipid droplets appeared. NMF is missing. After topical application of actives to RHE models, their distribution was characterized. The distribution maps reveal higher glycerol concentration in water-rich domains while OMC was concentrated in the lipid droplets.

The results show in how far the local constitution of skin triggers the local distribution of topically administered actives. The demonstrated Raman methodology can be used to understand penetration microroutes and to improve current skin models - in particular with respect to their barrier properties that should mimic the in vivo situation of human skin. 


\section{References}

'J Hadgraft, ME Lane. Skin, Phys Chem Chem Phys 13 (2011) 5215-22.

'GM O'Regan, PMJH Kemperman, A Sandilands, H Chen, LE Campbell, K Kroboth, R Watson, M Rowland, GJ Puppels, WHI McLean, PJ Caspers, AD Irvine, J Allergy Clin Immunol 126 (2010) 574-80.

${ }^{3}$ MO Visscher, GT Tolia, RR Wickett, SB Hoath, J Cosm Sci 54 (2003) 289-300.

${ }^{4} J A$ Bouwstra, PL Honeywell-Nguyen, GS Gooris, M Ponec, Prog Lipid Res (42) 2003 1-36.

${ }^{5}$ S Gregoire, C Ribaud, F Benech, JR Meunier, A Garrigues-Mazert, RH Guy, Brit J Dermatol 160 (2009) 8091.

${ }^{6}$ JW Wiechers, CL Kelly, TG Blease, JC Dederen, Int J Cosmetic Sci 26 (2004) 173-82.

7'M Foerster, MA Bolzinger, D Ach, G Montagnac, S Briançon, Pharm Res 28 (2011) 858-72.

${ }^{8}$ EW Smith, HI Maibach (eds.), Percutaneous penetration enhancers, $2^{\text {nd }}$ edn., Taylor \& Francis, Boca Raton London New York, 2006.

${ }^{9}$ European Union Reference Laboratory on Alternatives to Animal Testing, ECVAM, validation studies for skin irritation and skin corrosion tests.

${ }^{10}$ SkinEthic skin irritation test 42bis (using RHE model), standard operation protocol, 2009.

"S Gregoire, C Patouillet, C Noe, I Fossa, F Benech Kieffer, C Ribaud, Skin Pharmacol Physiol 21 (2008) 89-97.

${ }^{12}$ M Van Gele, B Geusens, L Brochez, R Speeckaert, J Lambert, Expert Opin Drug Deliv 8 (2011) 705-20.

${ }^{13}$ F Netzlaff, M Kaca, U Bock, E Haltner-Ukomadu, P Meiers, CM Lehr, UF Schaefer, Europ J Pharmac Biopharmac 66 (2007) 127-34.

${ }^{14} \mathrm{M}$ Schaefer-Korting, U Bock, W Diembeck, HJ Duesing, A Gamer, E Haltner-Ukimadu, C Hoffmann, M Kaca, H Kamp, S Kersen, M Kietzmann, HC Korting, HU Kraechter, CM Lehr, M Liebsch, A Mehling, C Mueller-Goymann, F Netzlaff, F Niedorf, MK Ruebbelke, U Schaefer, E Schmidt, S Schreiber, H Spielmann, A Vuia, M Weimer, ATLA 36 (2008) 161-87.

${ }^{15}$ JA Bouwstra, GS Gooris, A Weerheim, J Kempenaar, M Ponec, J Lipid Res 36 (1995) 496-504.

${ }^{16} \mathrm{~N}$ Alepee, C Tornier, C Robert, C Amsellem, MH Roux, O Doucet, J Pachot, M Meloni, A De Brugerolle de Fraissinette, Toxicology in Vitro 24 (2010) 257-64.

${ }^{17}$ A Gysler, B Kleuser, W Sippl, K Lange, HC Korting, HD Hoeltje, M Schaefer-Korting, J Pharma Res 16 (1999) 1386-91.

${ }^{18}$ FP Schmook, JG Meingasner, A Billich, Int J Pharm 215 (2001) 51-6.

${ }^{19}$ R Darlenski, S Sassing, N Tsankow, JW Fluhr, Europ J Pharmac Biopharmac 72 (2009) 295-303.

${ }^{20}$ HJ Weigmann, J Lademann, H Meffert, H Schaefer, W Sterry, Skin Pharmacol Appl Skin Physiol 12 (1999) 34-45.

${ }^{21}$ C Herkenne, A Naik, YN Kalia, J Hadgraft, RH Guy, J Invest Dermatol 127 (2007) 135-42. 
${ }^{22}$ H Wagner, KH Kostka, CM Lehr, UF Schaefer, Pharm Res 17 (2000) 1475-81.

${ }^{23}$ B Rozman, M Gasperlin, E Tinois-Tessoneaud, F Pirot, F Falson, Europ J Pharmac Biopharmac 72 (2009) 69-75.

${ }^{24}$ S Wachsmann-Hogiu, T Weeks, T Huser, Current Opinion Biotech 20 (2009) 63-73.

${ }^{25}$ PJ Caspers, GW Lucassen, R Wolthuis, HA Bruining, GJ Puppels, Biospectroscopy 8 (1998) 31-9.

${ }^{26}$ PJ Caspers, GW Lucassen, EA Carter, HA Bruining, GJ Puppels, J Invest Dermat 116 (2001) 434- 42.

${ }^{27}$ PJ Caspers, GW Lucassen, HA Bruining, GJ Puppels, J Raman Spectrosc 31 (2000) 813-8.

${ }^{28}$ PJ Caspers, GW Lucassen, GJ Puppels, Biophys J 85 (2003) 572-80.

${ }^{29} \mathrm{C}$ Adlhart, W Baschong, Int J Cosmetic Sci 33 (2011) 527-34.

${ }^{30}$ M Melot, PDA Pudney, AM Williamson, PJ Caspers, A Van der Pol, GJ Puppels, J Cont Release 138 (2009) 32-9.

${ }^{31}$ A Tfayli, O Piot, F Draux, F Pitre, M Manfait, Biopolymers 87 (2007) 261-73.

${ }^{32}$ S Pappinen, E Pryazhnikov, L Khiroug, MB Ericson, M Yliperttula, A Urtti, J Controll Release 161 (2012) 656-67.

${ }^{33}$ R Mendelsohn, CR Flach, DJ Moore, Biochim Biophys Acta 1758 (2006) 923-33.

${ }^{34} \mathrm{~J}$ Schulz, H Hohenberg, F Pfluecker, E Gaertner, T Will, S Pfeiffer, R Wepf, V Wendel, H Gers-Barlag, KP Wittern, Adv Drug Deliv Rev 54 (2002) 157-63.

${ }^{35}$ DD Verma, S Verma, G Blume, A Fahr, Int J Pharm 258 (2003) 141-51.

${ }^{36}$ JA Bouwstra, A De Graaff, GS Gooris, J Nijsse, JW Wiechers, AC Van Aelst, J Invest Dermatol 120 (2003) 750-8.

${ }^{37}$ RR Warner, MC Myers, DA Taylor, J Inv Dermatol 90 (1988) 218-24.

${ }^{38}$ SkinEthic, directions for use of reconstructed human epidermis (RHE). 2012.

${ }^{39}$ MM Jimenez, J Pelletier, MF Bobin, MC Martini, J Pharmaceut 272 (2004) 45-55.

${ }^{40}$ F Netzlaff, C-M Lehr, PW Wertz, UF Schaefer, Europ J Pharmac Biopharmac 60 (2005) 67-168. 


\section{Figure legends}

Fig. 1: Raman spectra of skin models close to the surface: untreated skin and skin treated with glycerol and OMC, respectively; compared to the Raman spectra of the pure compounds (averaged spectra). Some of the characteristic peaks of the pure actives are marked by arrows in the Raman spectra of treated skin.

Fig. 2: Virtual cross section of a skin model obtained by confocal Raman microspectroscopy: Distribution of water and lipids (left and middle). Histologic sections stained nile red for lipids (right). The typical thickness of the SC was 50 to $60 \mu \mathrm{m}$. Relative concentrations: bright color corresponds to high concentration, dark color to low concentration.

Fig. 3: Virtual cross section of a skin model obtained by confocal Raman microspectroscopy: Distribution of water, lipids and glycerol extracted from Raman measurements (measured from skin surface to depth through entire skin model). Relative concentrations: bright color corresponds to high concentration, dark color to low concentration.

Fig. 4: Virtual cross section of a skin model obtained by confocal Raman microspectroscopyscopy: Distribution of water, lipids and OMC extracted from Raman measurements (measured from skin surface to depth through entire skin model). Relative concentrations: bright color corresponds to high concentration, dark color to low concentration. Triangular marks indicate areas with high relative OMC and lipid concentration while the water concentration is low.

Fig. 5: Correlation of the fit coefficients for glycerol (left) and OMC (right) with the water coefficients in treated RHE models. 


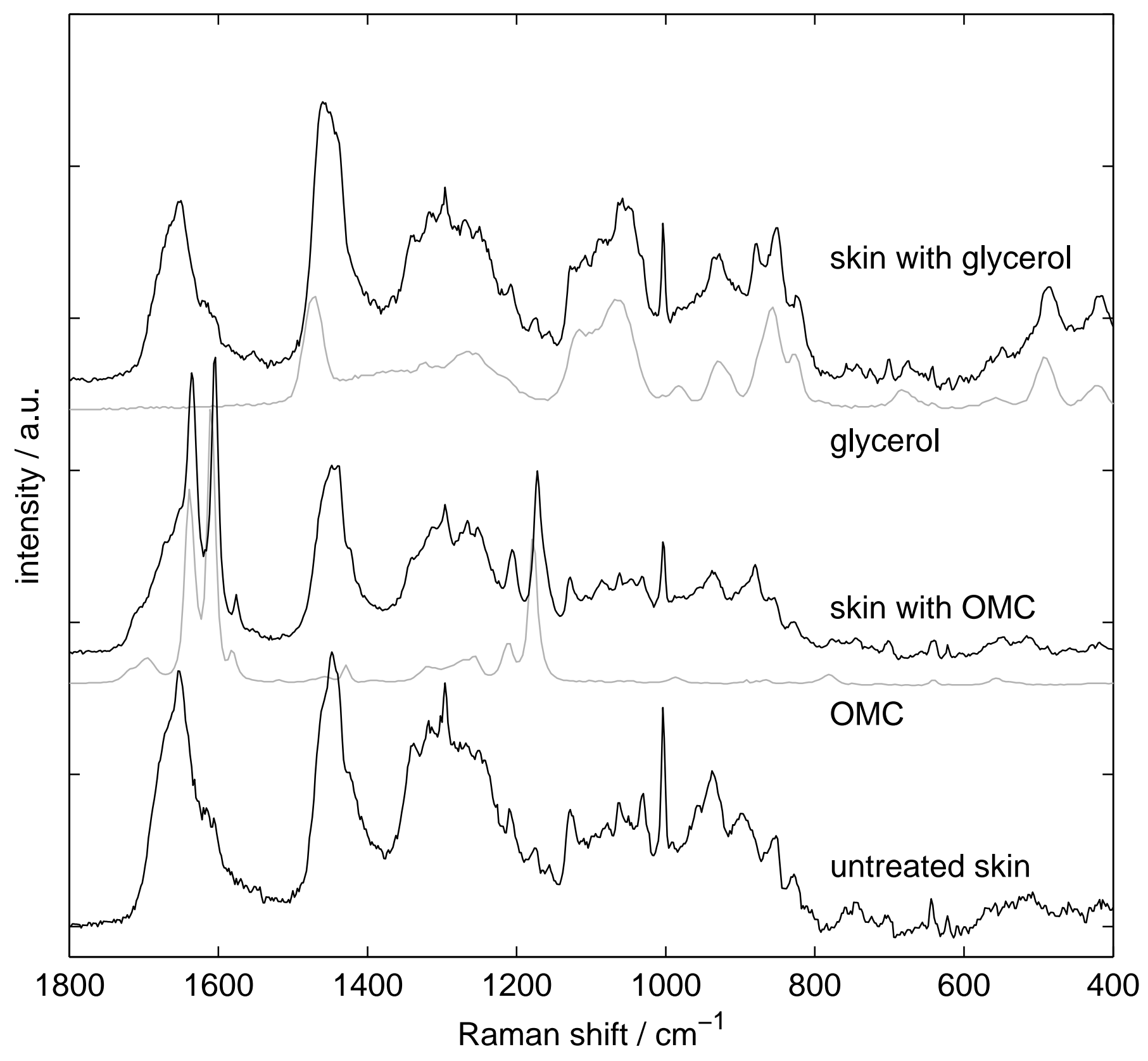


Click here to download high resolution image
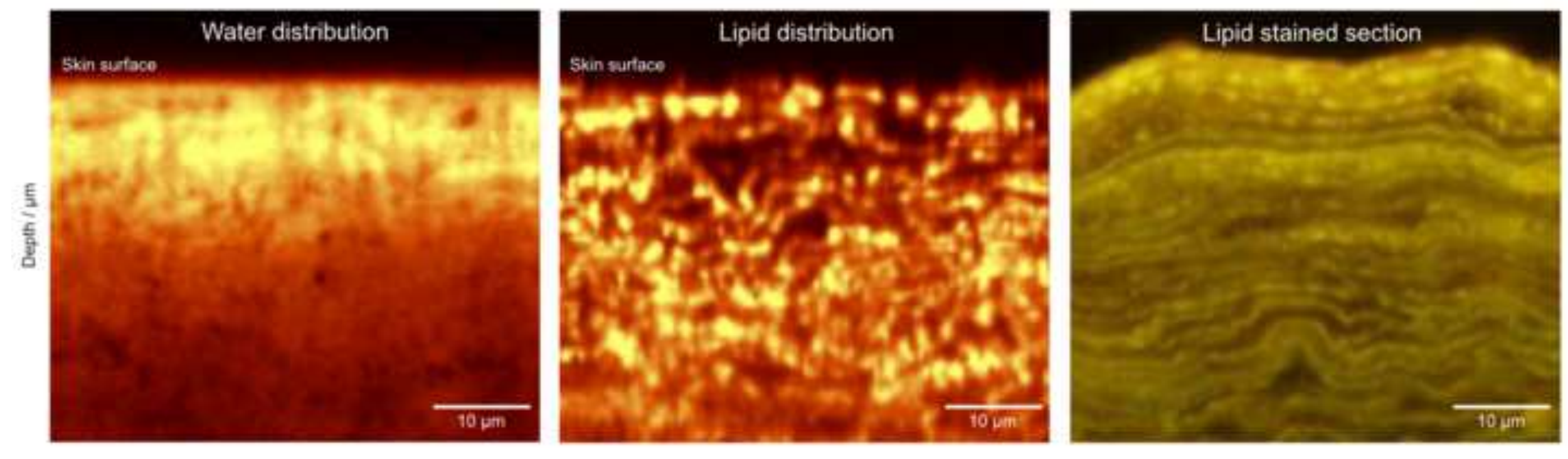

Clck here to download high resolution image

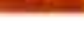


Figure 3
Click here to download high resolution image
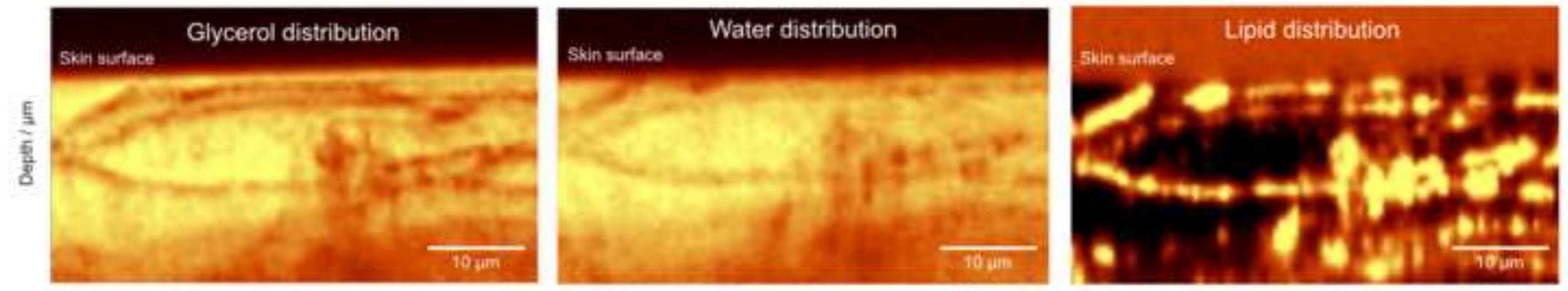


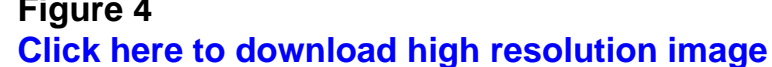
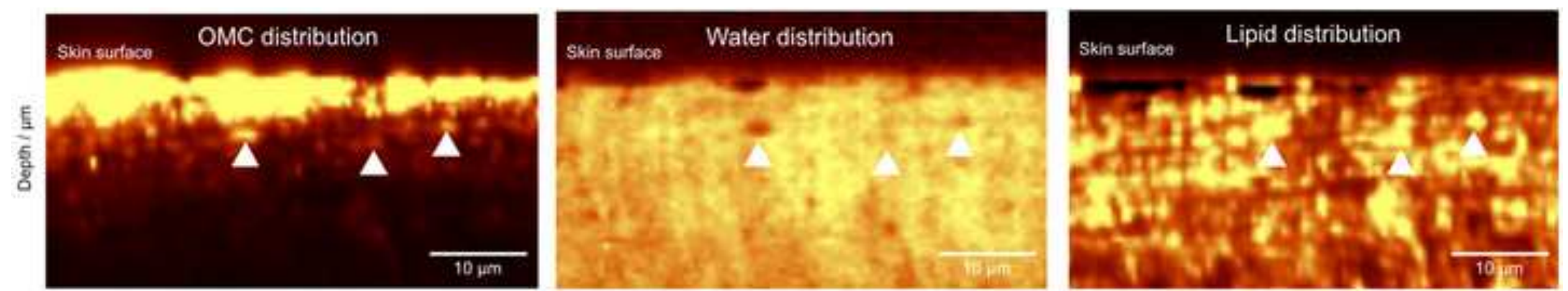
Figure 5
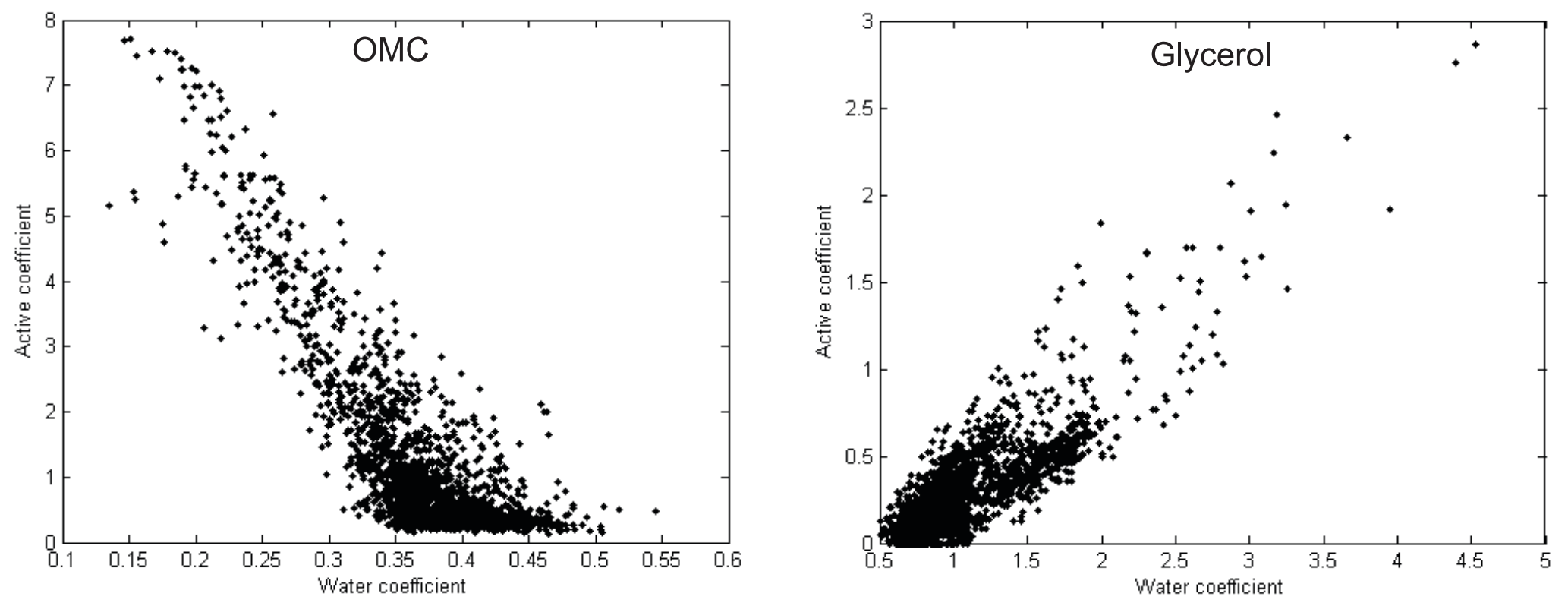